\title{
ERROR ANALYSIS FOR REGULARIZED MULTIDIMENSIONAL SAMPLING EXPANSIONS*
}

\author{
RASHAD M. ASHARABI ${ }^{\dagger}$ AND FATEMAH M. AL-ABBAS ${ }^{\dagger}$
}

\begin{abstract}
As it is known, the convergence rate of the multidimensional Whittaker-Kotelnikov-Shannon (WKS) sampling series is slow due to the slow decay of the sinc function. In this paper, we incorporate a convergence factor from the Bernstein space into the multidimensional WKS sampling series to establish regularized sampling and a corresponding improved convergence rate. The convergence rate of this regularized series depends on the decay of the convergence factor. Various bounds for the truncation of the regularized sampling series are investigated depending on the convergence factor. Furthermore, we estimate two types of perturbation errors associated with this series. Some numerical experiments are presented.
\end{abstract}

Key words. multidimensional sampling, error analysis, convergence rate

AMS subject classifications. 41A80, 32A05, 41A25, 41A30, 94A20

1. Introduction. Throughout this paper we use the parameters $\sigma>0,1 \leq p<\infty$, and variables $k:=\left(k_{1}, \ldots, k_{n}\right) \in \mathbb{Z}^{n}$ and $x:=\left(x_{1}, \ldots, x_{n}\right) \in \mathbb{R}^{n}$ unless stated otherwise. Denote by $L^{p}\left(\mathbb{R}^{n}\right)$ the Banach space of all complex-valued Lebesgue measurable functions of $n$ variables with the norm

$$
\|f\|_{p}=\left\{\begin{array}{lc}
\left(\int_{-\infty}^{\infty} \cdots \int_{-\infty}^{\infty}|f(x)|^{p} d x_{1} \ldots d x_{n}\right)^{1 / p}, & 1 \leq p<\infty, \\
{\operatorname{ess} \sup _{x \in \mathbb{R}^{n}}}|f(x)|, & p=\infty,
\end{array}\right.
$$

being finite. The Bernstein space $B_{\sigma}^{p}\left(\mathbb{R}^{n}\right)$ is the set of all functions from $L^{p}\left(\mathbb{R}^{n}\right)$ that can be extended to entire functions of exponential type $\sigma$. According to Schwartz's theorem [21, p. 109], it is defined by

$$
B_{\sigma}^{p}\left(\mathbb{R}^{n}\right)=\left\{f \in L^{p}\left(\mathbb{R}^{n}\right): \quad \operatorname{supp} \widehat{f} \subset[-\sigma, \sigma]^{n}\right\} .
$$

The multidimensional Whittaker-Kotelnikov-Shannon (WKS) sampling theorem states that if $f \in B_{\sigma}^{p}\left(\mathbb{R}^{n}\right)$, then $f$ can be represented as $[15,22,23,24]$

$$
f(x)=\sum_{k \in \mathbb{Z}^{n}} f(k h) \prod_{j=1}^{n} \operatorname{sinc}\left(\pi h^{-1} x_{j}-k_{j} \pi\right),
$$

where $k:=\left(k_{1}, \ldots, k_{n}\right) \in \mathbb{Z}^{n}, x:=\left(x_{1}, \ldots, x_{n}\right) \in \mathbb{R}^{n}$, and $h \in(0, \pi / \sigma]$. The sinc function is defined via

$$
\operatorname{sinc}(t):= \begin{cases}\frac{\sin t}{t}, & t \neq 0 \\ 1, & t=0 .\end{cases}
$$

The series (1.2) converges absolutely and uniformly on $\mathbb{R}^{n}$ and uniformly on any compact subset of $\mathbb{C}^{n}$; see, e.g., [24]. The convergence of this series is slow unless $\left|f\left(x_{1}, \ldots, x_{n}\right)\right|$

*Received April 1, 2020. Accepted May 16, 2020. Published online on June 25, 2020. Recommended by Frank Stenger.

${ }^{\dagger}$ Department of Mathematics, College of Arts and Sciences, Najran University, Najran, Saudi Arabia, ( $\{$ rashad1974@hotmail.com, alfatemah760@hotmail.com\}). 
decays rapidly as $\left|x_{j}\right| \rightarrow \infty$ for all $1 \leq j \leq n$, and it is of the order of $O\left(N^{1 / p}\right), p>1$; cf. [25]. The slow convergence rate is due to the slow decay of the sinc function. Bounds of truncation and aliasing errors for the multidimensional sampling series (1.2) have been investigated in the literature; cf., e.g., [7, 13, 18, 20, 24, 25, 26]. Ye established a bound for the truncation error associated with the series (1.2) based on localized sampling without a decay assumption on $f$; cf. [25]. To the best of our knowledge, there are no studies on the perturbation error associated with the series in (1.2). We would like to mention here that the error analysis for uniform and nonuniform univariate sampling series has been extensively studied (see, e.g., $[1,3,6,8,10,17,19,27]$ and the references therein), while the multidimensional sampling did not take that space of interest.

The aim of this paper is to incorporate a convergence factor, from the Bernstein space $B_{r}^{p}\left(\mathbb{R}^{n}\right), r>0$, into the series at the right-hand side of (1.2). The convergence rate will be improved, and it is mainly dependent on the decay of the convergence factor. We investigate bounds for two types of truncation errors associated with the regularized series, and the decay of those bound will be dependent on the convergence factor. Two bounds for the perturbation errors, amplitude and jitter, associated with this series will be estimated. Here we would like to point out that there are many studies on the perturbation errors associated with various types of one-dimensional sampling series (see, e.g., $[1,2,3,9]$ ), while no single study, to the best of our knowledge, has been carried out for the estimation of the amplitude and jitter errors associated with multidimensional sampling series.

The rest of the paper is organized as follows: the next section outlines preliminary results which will be used in the sequel. Section 3 is devoted to the regularized multidimensional sampling series, and two types of truncation errors for this sampling series are investigated. In Section 4, we investigate perturbation errors associated with this type of sampling expansion. Section 5 deals with numerical illustrations.

2. Preliminaries. This section is devoted to five preliminary results that will be used thereafter in the next sections. The proofs of these results are not difficult and depend on some results of sampling theory. We start with the following two lemmas:

LEMMA 2.1. If $p, q>1$ such that $1 / p+1 / q=1$, then for any $t \in \mathbb{R}$ we have

$$
\left(\sum_{\ell=-\infty}^{\infty}\left|\varphi\left(h^{-1} t-\ell\right) \operatorname{sinc}\left(\pi h^{-1} t-\ell \pi\right)\right|^{q}\right)^{1 / q} \leq p\|\varphi\|_{\infty}
$$

where $\varphi \in B_{\sigma}^{\infty}(\mathbb{R})$ and $\|\varphi\|_{\infty}$ is defined in $(1.1)$.

Proof. From the definition of the function $\varphi$, we have

$$
\begin{aligned}
\left(\sum_{\ell=-\infty}^{\infty}\left|\varphi\left(h^{-1} t-\ell\right) \operatorname{sinc}\left(\pi h^{-1} t-\ell \pi\right)\right|^{q}\right)^{1 / q} & \\
& \leq\|\varphi\|_{\infty}\left(\sum_{\ell=-\infty}^{\infty}\left|\operatorname{sinc}\left(\pi h^{-1} t-\ell \pi\right)\right|^{q}\right)^{1 / q}
\end{aligned}
$$

Now we state an inequality of Splettstösser et al.; cf. [9],

$$
\left(\sum_{\ell=-\infty}^{\infty}\left|\operatorname{sinc}\left(\pi h^{-1} t-\ell \pi\right)\right|^{q}\right)^{1 / q} \leq p
$$

Combining (2.3) and (2.2) gives (2.1). 
Lemma 2.2. Assume $p, q>1$ such that $1 / p+1 / q=1$. For any $t \in \mathbb{R}, N \in \mathbb{N}$, we have

$$
\left(\sum_{\left|h^{-1} t-\ell\right|>N}\left|\varphi\left(h^{-1} t-\ell\right) \operatorname{sinc}\left(\pi h^{-1} t-\ell \pi\right)\right|^{q}\right)^{1 / q} \leq C_{p} \frac{\alpha(N)}{N^{1 / p}},
$$

where $\alpha$ is a non-negative decreasing function on $(0, \infty]$ that satisfies $|\varphi(t)| \leq \alpha(|t|)$ on $(0, \infty]$. The constant $C_{p}$ is given by

$$
C_{p}:=\frac{1}{\pi}\left(\frac{2}{q-1}\right)^{1 / q}
$$

Proof. Define $g(\cdot)$ by

$$
g(t):=\sum_{\left|h^{-1} t-\ell\right|>N}\left|\varphi\left(h^{-1} t-\ell\right) \operatorname{sinc}\left(\pi h^{-1} t-\ell \pi\right)\right|^{q} .
$$

This function is periodic with period $h$. Indeed, we have

$$
\begin{aligned}
g(t+h) & :=\sum_{\left|h^{-1}(t+h)-\ell\right|>N}\left|\varphi\left(h^{-1}(t+h)-\ell\right) \operatorname{sinc}\left(\pi h^{-1}(t+h)-\ell \pi\right)\right|^{q} \\
& =\sum_{\left|h^{-1} t-(\ell-1)\right|>N}\left|\varphi\left(h^{-1} t-(\ell-1)\right) \operatorname{sinc}\left(\pi h^{-1} t-(\ell-1) \pi\right)\right|^{q} .
\end{aligned}
$$

Replacing $\ell-1$ by $\ell$ in (2.7) proves the $h$-periodicity of (2.6). Now, we prove the lemma only on the interval $[-h / 2, h / 2]$. Since $|\varphi(t)| \leq \alpha(|t|)$, we have

$$
\left|\varphi\left(h^{-1} t-\ell\right)\right| \leq \alpha\left(\left|h^{-1} t-\ell\right|\right) \leq \alpha(N),
$$

for all $t$ satisfying $\left|h^{-1} t-\ell\right|>N$. Therefore,

$$
\begin{gathered}
\left(\sum_{\left|h^{-1} t-\ell\right|>N}\left|\varphi\left(h^{-1} t-\ell\right) \operatorname{sinc}\left(\pi h^{-1} t-\ell \pi\right)\right|^{q}\right)^{1 / q} \\
\leq \alpha(N)\left(\sum_{\left|h^{-1} t-\ell\right|>N} \frac{1}{\left|\pi h^{-1} t-\ell \pi\right|^{q}}\right)^{1 / q} \leq \frac{\alpha(N)}{\pi}\left(2 \sum_{n=N}^{\infty} \frac{1}{n^{q}}\right)^{1 / q} \\
\leq \frac{\alpha(N)}{\pi}\left(2 \int_{N}^{\infty} \frac{d s}{s^{q}}\right)^{1 / q}=\frac{C_{p} \alpha(N)}{N^{1 / p}} .
\end{gathered}
$$

For $x=\left(x_{1}, \ldots, x_{n}\right) \in \mathbb{R}^{n}$, we define $\mathbb{Z}_{N}^{n}(x)$ as

$$
\mathbb{Z}_{N}^{n}(x):=\left\{k \in \mathbb{Z}^{n}:-N<\pi h^{-1} x_{j}-k_{j} \leq N, \quad 1 \leq j \leq n\right\} .
$$

In the following result, we establish an estimate for infinite sums of $n$-products of sinc functions. This bound will be used in the proof of Theorem 3.3.

Lemma 2.3. Let $p, q>1$ such that $1 / p+1 / q=1$ and $\left\{\varphi_{j}\right\}_{j=1}^{n} \in B_{\sigma}^{\infty}(\mathbb{R})$. Then, for $x \in \mathbb{R}^{n}$, we have

$$
\begin{aligned}
\left(\sum_{k \notin \mathbb{Z}_{N}^{n}(x)}\left|\prod_{j=1}^{n} \varphi_{j}\left(h^{-1} x_{j}-k_{j}\right) \operatorname{sinc}\left(\pi h^{-1} x_{j}-k_{j} \pi\right)\right|^{q}\right)^{1 / q} \\
\leq p^{n-1} \frac{C_{p}}{N^{1 / p}} \sum_{\ell=1}^{n} \alpha_{\ell}(N) \prod_{j=1, j \neq \ell}^{n}\left\|\varphi_{j}\right\|_{\infty},
\end{aligned}
$$


where $N \in \mathbb{N}, C_{p}$ is defined in (2.5), and $\alpha_{j}$ is a non-negative and strictly decreasing function on $(0, \infty)$ which satisfies

$$
\left|\varphi_{j}\left(x_{j}\right)\right| \leq \alpha_{j}\left(\left|x_{j}\right|\right), \quad 1 \leq j \leq n .
$$

Proof. In view of the definition of $\mathbb{Z}_{N}^{n}(x)$, we obtain

$$
\begin{aligned}
& \left(\sum_{k \notin \mathbb{Z}_{N}^{n}(x)}\left|\prod_{j=1}^{n} \varphi_{j}\left(h^{-1} x_{j}-k_{j}\right) \operatorname{sinc}\left(\pi h^{-1} x_{j}-k_{j} \pi\right)\right|^{q}\right)^{1 / q} \\
& \quad \leq\left(\sum_{\ell=1}^{n} \sum_{k \notin \mathbb{Z}_{N}^{n}\left(x_{\ell}\right)} \prod_{j=1}^{n}\left|\varphi_{j}\left(h^{-1} x_{j}-k_{j}\right) \operatorname{sinc}\left(\pi h^{-1} x_{j}-k_{j} \pi\right)\right|^{q}\right)^{1 / q},
\end{aligned}
$$

where $\mathrm{Z}_{N}^{n}\left(x_{\ell}\right)$ is defined by

$$
\mathrm{Z}_{N}^{n}\left(x_{\ell}\right):=\left\{k \in \mathbb{Z}^{n}:-N<\pi h^{-1} x_{\ell}-k_{\ell} \leq N\right\} .
$$

Since $\left\{\varphi_{j}\right\}_{j=1}^{n} \in B_{\sigma}^{\infty}(\mathbb{R})$, we have

$$
\left|\varphi_{j}\left(x_{j}\right)\right| \leq\left\|\varphi_{j}\right\|_{\infty} .
$$

From the assumption in (2.10), we have

$$
\left|\varphi_{\ell}\left(h^{-1} x_{\ell}-k_{\ell}\right)\right| \leq \alpha_{\ell}\left(\left|h^{-1} x_{\ell}-k_{\ell}\right|\right) \leq \alpha_{\ell}(N),
$$

for all $x_{\ell}$ satisfying $\left|h^{-1} x_{\ell}-k_{\ell}\right|>N, 1 \leq \ell \leq n$. Combining (2.13), (2.12), (2.3), (2.4), and (2.11) gives (2.9).

In the following lemma, we will estimate infinite sums of samples of Bernstein functions that satisfy a decay condition. This result will be used in the proof of Theorem 3.3. For a fixed $\ell$, we define the symbol $\mathbb{Z}_{\ell, N}^{n}$ to be

$$
\mathbb{Z}_{\ell, N}^{n}=\left\{k \in \mathbb{Z}^{n}:\left|k_{\ell}\right|>N\right\} .
$$

LemMA 2.4. Let $f \in B_{\sigma}^{2}\left(\mathbb{R}^{n}\right)$ satisfy a decay condition of the form

$$
x_{\ell}^{m_{\ell}} f(x) \in L^{2}\left(\mathbb{R}^{n}\right),
$$

where $1 \leq \ell \leq n$ and $m_{\ell}$ is a non-negative integer. Then we have

$$
\sum_{k \in \mathbb{Z}_{\ell, N}^{n}}|f(k h)|^{2} \leq \frac{2 E_{m_{\ell}}^{2}}{h^{2 m_{\ell}+n}(N+1)^{2 m_{\ell}}\left(1-4^{-m_{\ell}}\right)},
$$

where $\mathbb{Z}_{\ell, N}^{n}$ is defined in (2.14) and

$$
E_{m_{\ell}}^{2}=\int_{-\infty}^{\infty} \ldots \int_{-\infty}^{\infty}\left|x_{\ell}^{m_{\ell}} f(x)\right|^{2} d x_{1} \ldots d x_{n}
$$

for any $1 \leq \ell \leq n$.

Proof. Since $f \in B_{\sigma}^{2}\left(\mathbb{R}^{n}\right)$, the function $x_{\ell}^{m_{\ell}} f(x)$ is an entire function of exponential type $\sigma$; cf., e.g., [21, Chpater 3]. Therefore, $x_{\ell}^{m_{\ell}} f(x) \in B_{\sigma}^{2}\left(\mathbb{R}^{n}\right)$ because of the assumption 
$x_{\ell}^{m_{\ell}} f(x) \in L^{2}\left(\mathbb{R}^{n}\right)$. Applying the multidimensional Parseval's identity for the function $x_{\ell}^{m_{\ell}} f(x)$, cf., e.g., [16, p. 158], implies

$$
\sum_{k \in \mathbb{Z}^{n}} k_{\ell}^{2 m_{\ell}}|f(k h)|^{2}=\frac{E_{m_{\ell}}^{2}}{h^{2 m_{\ell}+n}}
$$

where $E_{m_{\ell}}^{2}$ is defined above. For a fixed $\ell \in \mathbb{N}$, we truncate the $\ell$-th sum to get

$$
\sum_{k_{1}=-\infty}^{\infty} \ldots \sum_{k_{\ell}=\tau}^{2 \tau-1} \ldots \sum_{k_{n}=-\infty}^{\infty} k_{\ell}^{2 m_{\ell}}|f(k h)|^{2} \leq \frac{E_{m_{\ell}}^{2}}{h^{2 m_{\ell}+n}}
$$

and therefore

$$
\begin{aligned}
& \tau^{2 m_{\ell}} \sum_{k_{1}=-\infty}^{\infty} \ldots \sum_{k_{\ell}=\tau}^{2 \tau-1} \ldots \sum_{k_{n}=-\infty}^{\infty}|f(k h)|^{2} \\
& \leq \sum_{k_{1}=-\infty}^{\infty} \ldots \sum_{k_{\ell}=\tau}^{2 \tau-1} \ldots \sum_{k_{n}=-\infty}^{\infty} k_{\ell}^{2 m_{\ell}}|f(k h)|^{2} .
\end{aligned}
$$

Combining (2.18) and (2.19) gives

$$
\sum_{k_{1}=-\infty}^{\infty} \ldots \sum_{k_{\ell}=\tau}^{2 \tau-1} \ldots \sum_{k_{n}=-\infty}^{\infty}|f(k h)|^{2} \leq \frac{E_{m_{\ell}}^{2}}{h^{2 m_{\ell}+n} \tau^{2 m_{\ell}}}
$$

Putting $\tau=2^{r}(N+1)$ in (2.20) and taking the sum from $r=0$ up to $\infty$, we obtain

$$
\sum_{k_{1}=-\infty}^{\infty} \ldots \sum_{k_{\ell}>N} \ldots \sum_{k_{n}=-\infty}^{\infty}|f(k h)|^{2} \leq \frac{E_{m_{\ell}}^{2}}{h^{2 m_{\ell}+n}(N+1)^{2 m_{\ell}}\left(1-4^{\left.-m_{\ell}\right)}\right.}
$$

which is (2.16).

At the end of this section, we introduce the following result, which is required to prove Theorem 3.4:

LEMMA 2.5. Under the assumptions of Lemma 2.3, we have for a fixed $\ell$,

$$
\begin{array}{r}
\left(\sum_{k \in \mathbb{Z}_{\ell, N}^{n}} \prod_{j=1}^{n}\left|\varphi_{j}\left(h^{-1} x_{j}-k_{j}\right) \operatorname{sinc}\left(\pi h^{-1} x_{j}-k_{j} \pi\right)\right|^{q}\right)^{1 / q} \\
\leq p^{n} \alpha_{\ell}(N) \prod_{j=1, j \neq \ell}^{n}\left\|\varphi_{j}\right\|_{\infty},
\end{array}
$$

where $\mathbb{Z}_{\ell, N}^{n}$ is defined in (2.14). 
Proof. In view of the definition of $\mathbb{Z}_{\ell, N}^{n}$ and using inequality (2.1), we obtain

$$
\begin{aligned}
\left(\sum_{k \in \mathbb{Z}_{\ell, N}^{n}} \prod_{j=1}^{n}\left|\varphi_{j}\left(h^{-1} x_{j}-k_{j}\right) \operatorname{sinc}\left(\pi h^{-1} x_{j}-k_{j} \pi\right)\right|^{q}\right)^{1 / q} & \\
\leq & \left(\sum_{\left|k_{\ell}\right| \geq N}\left|\varphi_{\ell}\left(h^{-1} x_{\ell}-k_{\ell}\right) \operatorname{sinc}\left(\pi h^{-1} x_{\ell}-k_{\ell} \pi\right)\right|^{q}\right)^{1 / q} \\
& \times \prod_{j=1, j \neq \ell}^{n}\left(\sum_{k_{j}=-\infty}^{\infty}\left|\varphi_{j}\left(h^{-1} x_{j}-k_{j}\right) \operatorname{sinc}\left(\pi h^{-1} x_{j}-k_{j} \pi\right)\right|^{q}\right)^{1 / q} \\
= & p^{n-1} \prod_{j=1, j \neq \ell}^{n}\left\|\varphi_{j}\right\|_{\infty}\left(\sum_{\left|k_{\ell}\right| \geq N}\left|\varphi_{\ell}\left(h^{-1} x_{\ell}-k_{\ell}\right) \operatorname{sinc}\left(\pi h^{-1} x_{\ell}-k_{\ell} \pi\right)\right|^{q}\right)^{1 / q} .
\end{aligned}
$$

The assumption in (2.10) gives

$$
\left|\varphi_{\ell}\left(h^{-1} x_{\ell}-k_{\ell}\right)\right| \leq \alpha_{\ell}(N),
$$

for all $\left|x_{\ell}\right|<N h, 1 \leq \ell \leq n$. Using (2.23) and (2.3) implies

$$
\left(\sum_{\left|k_{\ell}\right| \geq N}\left|\varphi_{\ell}\left(h^{-1} x_{\ell}-k_{\ell}\right) \operatorname{sinc}\left(\pi h^{-1} x_{\ell}-k_{\ell} \pi\right)\right|^{q}\right)^{1 / q} \leq p \alpha_{\ell}(N) .
$$

Combining (2.24) and (2.22) gives (2.21).

3. Regularized sampling and truncation error bounds. In this section, we incorporate a convergence factor from the Bernstein space $B_{\theta \sigma}^{p}\left(\mathbb{R}^{n}\right), 0<\theta<1$, into (1.2) to define the regularized multidimensional sampling series (3.1). The speed of the convergence of the regularized sampling series is determined by the decay of the convergence factor. Two types of truncation errors for the regularized sampling series are investigated, and the bounds of those are dependent on the convergence factor. From now on, unless stated otherwise, $h \in(0, \pi / \sigma]$ and $0<\theta<1$.

THEOREM 3.1. Let $f \in B_{(1-\theta) \sigma}^{p}\left(\mathbb{R}^{n}\right), 0<\theta<1$, and $\prod_{j=1}^{n} \varphi_{j} \in B_{\theta \sigma}^{p}\left(\mathbb{R}^{n}\right)$. Then, for $x \in \mathbb{R}^{n}$, we have the regularized multidimensional sampling expansion

$$
f(x)=\sum_{k \in \mathbb{Z}^{n}} f(k h) \prod_{j=1}^{n} \varphi_{j}\left(h^{-1} x_{j}-k_{j}\right) \operatorname{sinc}\left(\pi h^{-1} x_{j}-k_{j} \pi\right),
$$

where $\varphi_{j}(0)=1, j=1, \ldots, n$. The series (3.1) converges absolutely and uniformly on $\mathbb{R}^{n}$ and on any compact subset of $\mathbb{C}^{n}$.

Proof. Since $\varphi_{j} \in B_{\theta \sigma}^{p}\left(\mathbb{R}^{n}\right)$ and $f \in B_{(1-\theta) \sigma}^{p}\left(\mathbb{R}^{n}\right)$, for any fixed $x \in \mathbb{R}^{n}$, the function

$$
x \rightarrow f(x) \prod_{j=1}^{n} \varphi_{j}\left(h^{-1}\left(s_{j}-x_{j}\right)\right)
$$

belong to $B_{\sigma}^{p}\left(\mathbb{R}^{n}\right)$. Apply the multidimensional sampling expansion in equation (1.2) with $h \in(0,(1-\theta)]$ to obtain

$$
f(x) \prod_{j=1}^{n} \varphi_{j}\left(h^{-1}\left(s_{j}-x_{j}\right)=\sum_{k \in \mathbb{Z}^{n}} f(k h) \prod_{j=1}^{n} \varphi_{j}\left(h^{-1} s_{j}-k_{j}\right) \operatorname{sinc}\left(\pi h^{-1} s_{j}-k_{j} \pi\right) .\right.
$$


Substituting $s_{j}=x_{j}$ and using the assumption $\varphi_{j}(0)=1, j=1, \ldots, n$, we get (3.1). The convergence of the series in (3.1) follows from the convergence of (1.2).

REMARK 3.2. Jagerman in [17] has established a special case of the series (3.1) for functions of one variable, and his convergence factor was $\varphi(t)=\operatorname{sinc}^{m}(\theta \pi t / m), m \in \mathbb{N}$. The convergence rate of this factor is of the order of $O\left(1 / N^{m}\right)$. This type of regularized sampling has been used in approximating the eigenvalues of boundary value problems; cf., e.g., $[5,11]$.

In the following results, we investigate bounds for two types of truncation errors associated with the series (3.1). The first one is the classical truncation error, and it will be studied with various decay conditions. The second one is based on localized sampling without a decay condition.

Define the set $\mathbb{B}_{N}^{n}$ to be

$$
\mathbb{B}_{N}^{n}:=\left\{k \in \mathbb{Z}^{n}:\left|k_{j}\right| \leq N \quad \forall j=1, \ldots, n\right\} .
$$

The truncation error associated with the series in (3.1) is defined as for all $x \in \mathbb{R}^{n}$ as

$$
\begin{aligned}
T_{N}(x): & =f(x)-\sum_{k \in \mathbb{B}_{N}^{n}} f(k h) \prod_{j=1}^{n} \varphi_{j}\left(h^{-1} x_{j}-k_{j}\right) \operatorname{sinc}\left(\pi h^{-1} x_{j}-k_{j} \pi\right) \\
& =\sum_{k \in \mathbb{Z}^{n} / \mathbb{B}_{N}^{n}} f(k h) \prod_{j=1}^{n} \varphi_{j}\left(h^{-1} x_{j}-k_{j}\right) \operatorname{sinc}\left(\pi h^{-1} x_{j}-k_{j} \pi\right) .
\end{aligned}
$$

It is not hard to see that $\mathbb{Z}^{n} / \mathbb{B}_{N}^{n} \subseteq \bigcup_{\ell=1}^{n} \mathbb{Z}_{\ell, N}^{n}$, and then

$$
\begin{aligned}
\sum_{k \in \mathbb{Z}^{n} / \mathbb{B}_{N}^{n}}\left|f(k h) \prod_{j=1}^{n} \varphi_{j}\left(h^{-1} x_{j}-k_{j}\right) \operatorname{sinc}\left(\pi h^{-1} x_{j}-k_{j} \pi\right)\right| \\
\quad \leq \sum_{\ell=1}^{n} \sum_{k \in \mathbb{Z}_{\ell, N}^{n}}|f(k h)| \prod_{j=1}^{n}\left|\varphi_{j}\left(h^{-1} x_{j}-k_{j}\right) \operatorname{sinc}\left(\pi h^{-1} x_{j}-k_{j} \pi\right)\right|,
\end{aligned}
$$

where $\mathbb{Z}_{\ell, N}^{n}$ is defined in (2.14). Therefore

$$
\left|T_{N}(x)\right| \leq \sum_{\ell=1}^{n} \sum_{k \in \mathbb{Z}_{\ell, N}^{n}}|f(k h)| \prod_{j=1}^{n}\left|\varphi_{j}\left(h^{-1} x_{j}-k_{j}\right) \operatorname{sinc}\left(\pi h^{-1} x_{j}-k_{j} \pi\right)\right| .
$$

THEOREM 3.3. Let $f \in B_{(1-\theta) \sigma}^{2}\left(\mathbb{R}^{n}\right)$ satisfies the decay condition (2.15). Then, for $x \in \mathbb{R}^{n},\left|x_{j}\right|<N h, 1 \leq j \leq n$, we have the following pointwise bound:

$$
\left|T_{N}(x)\right| \leq \sum_{\ell=1}^{n} \frac{\xi_{\ell, m_{\ell}} \alpha_{\ell}(N)}{\sqrt{\pi}(N+1)^{m_{\ell}}}\left\{\frac{\left|\sin \left(\pi h^{-1} x_{\ell}\right)\right|}{\sqrt{N \pi-\pi h^{-1} x_{\ell}}}+\frac{\left|\sin \left(\pi h^{-1} x_{\ell}\right)\right|}{\sqrt{N \pi+\pi h^{-1} x_{\ell}}}\right\} \prod_{j=1, j \neq \ell}^{n}\left\|\varphi_{j}\right\|_{\infty},
$$

where $\xi_{\ell, m_{\ell}}:=\frac{2^{n-1 / 2} E_{m_{\ell}}}{h^{m_{\ell}+n / 2} \sqrt{1-4^{-m_{j}}}}$, and $C_{p}, \alpha_{j}$, and $E_{m_{\ell}}$ are given in (2.5), (2.10), and (2.17), respectively.

Proof. Since $f \in B_{(1-\theta) \sigma}^{2}\left(\mathbb{R}^{n}\right)$, the regularized multidimensional sampling expansion (3.1) holds. Applying the generalized Cauchy-Schwartz inequality to the left-hand side 
of (3.5) implies

$$
\begin{aligned}
\left|T_{N}(x)\right| \leq \sum_{\ell=1}^{n}\left(\sum_{k \in \mathbb{Z}_{\ell, N}^{n}}|f(k h)|^{2}\right)^{1 / 2} & \\
& \quad \times\left(\sum_{k \in \mathbb{Z}_{\ell, N}^{n}} \prod_{j=1}^{n}\left|\varphi_{j}\left(h^{-1} x_{j}-k_{j}\right) \operatorname{sinc}\left(\pi h^{-1} x_{j}-k_{j} \pi\right)\right|^{2}\right)^{1 / 2} .
\end{aligned}
$$

For a fixed $\ell$ and by using the inequality (2.1), we have

$$
\begin{aligned}
& \left(\sum_{k \in \mathbb{Z}_{\ell, N}^{n}} \prod_{j=1}^{n}\left|\varphi_{j}\left(h^{-1} x_{j}-k_{j}\right) \operatorname{sinc}\left(\pi h^{-1} x_{j}-k_{j} \pi\right)\right|^{2}\right)^{1 / 2} \\
& \quad \leq 2^{n-1} \prod_{j=1, j \neq \ell}^{n}\left\|\varphi_{j}\right\|_{\infty}\left(\sum_{\left|k_{\ell}\right|>N}\left|\varphi_{\ell}\left(h^{-1} x_{\ell}-k_{\ell}\right) \operatorname{sinc}\left(\pi h^{-1} x_{\ell}-k_{\ell} \pi\right)\right|^{2}\right)^{1 / 2}
\end{aligned}
$$

The assumption in (2.10) gives (2.23). Under the assumption $\left|t_{\ell}\right|<N h$, Jagerman has established the following inequality, cf. [17, p. 716]:

$$
\begin{aligned}
& \left(\sum_{\left|k_{\ell}\right|>N}\left|\operatorname{sinc}\left(\pi h^{-1} x_{\ell}-k_{\ell} \pi\right)\right|^{2}\right)^{1 / 2} \\
& \quad \leq \frac{\left|\sin \left(\pi h^{-1} x_{\ell}\right)\right|}{\sqrt{\pi}}\left\{\frac{1}{\sqrt{N \pi-\pi h^{-1} x_{\ell}}}+\frac{1}{\sqrt{N \pi+\pi h^{-1} x_{\ell}}}\right\} .
\end{aligned}
$$

Combining (3.8), (2.23), (3.7), and (2.16) gives (3.6), and the proof is completed.

The decay of the pointwise bound in (3.6) depends on the convergence factor $\varphi_{\ell}$, $\ell=1, \ldots, n$, and it is of order $O\left(\sum_{\ell=1}^{n} \alpha_{\ell}(N) / N^{m_{\ell}+1 / 2}\right)$ as $N \rightarrow \infty$. If we apply the last processing to the WKS sampling series (1.2), then the decay of the pointwise bound will be of order $O\left(\sum_{\ell=1}^{n} N^{-m_{\ell}-1 / 2}\right)$ as $N \rightarrow \infty$. This shows the importance of incorporating the convergence factor $\varphi_{\ell}$ into the series (1.2).

The next theorem gives a uniform bound for the truncation error defined in (3.3) using a decay condition of the form

$$
|f(x)| \leq \frac{A}{\prod_{j=1}^{n}\left(1+\left|x_{j}\right|^{\alpha+1}\right)},
$$

where $x \in \mathbb{R}^{n}$ and $A, \alpha$ are non-negative real numbers.

THEOREM 3.4. If $f \in B_{(1-\theta) \sigma}^{p}\left(\mathbb{R}^{n}\right)$ satisfies the decay condition (3.9), then we have the following uniform bound:

$$
\left|T_{N}(x)\right| \leq \frac{A 2^{1 / p} p^{n} D^{n-1}(\alpha)}{h^{\alpha+1}((\alpha+1) p-1)^{1 / p} N^{\alpha+1-1 / p}} \sum_{\ell=1}^{n} \alpha_{\ell}(N) \prod_{j=1, j \neq \ell}^{n}\left\|\varphi_{j}\right\|_{\infty},
$$

where

$$
\mathcal{D}(t)=\left(1+\frac{2}{h(t+1)} \mathcal{B}\left(\frac{1}{t+1}, p-\frac{1}{t+1}\right)\right)^{1 / p}
$$


and $\mathcal{B}$ is the Beta function.

Proof. Since $f \in B_{(1-\theta) \sigma}^{p}\left(\mathbb{R}^{n}\right)$, the regularized series expansion (3.1) holds. Applying the generalized Hölder inequality to the left-hand side of (3.5), we obtain

$$
\begin{aligned}
\left|T_{N}(x)\right| \leq \sum_{\ell=1}^{n}( & \left.\sum_{k \in \mathbb{Z}_{\ell, N}^{n}}|f(k h)|^{p}\right)^{1 / p} \\
& \times\left(\sum_{k \in \mathbb{Z}_{\ell, N}^{n}} \prod_{j=1}^{n}\left|\varphi_{j}\left(h^{-1} x_{j}-k_{j}\right) \operatorname{sinc}\left(\pi h^{-1} x_{j}-k_{j} \pi\right)\right|^{q}\right)^{1 / q},
\end{aligned}
$$

where $p, q>1$ and $1 / p+1 / q=1$. Since $f$ satisfies the decay condition (3.9), we obtain

$$
\sum_{k \in \mathbb{Z}_{\ell, N}^{n}}|f(k h)|^{p} \leq A^{p} \sum_{k \in \mathbb{Z}_{\ell, N}^{n}} \prod_{j=1}^{n} \frac{1}{\left(1+\left|k_{j} h\right|^{\alpha+1}\right)^{p}}
$$

where $\ell$ is fixed. For $p>1$ and $\alpha>0$, the authors of [7] have established the following inequalities:

$$
\sum_{\nu=-\infty}^{\infty} \frac{1}{\left(1+|\nu h|^{\alpha+1}\right)^{p}} \leq 1+\frac{2}{h(\alpha+1)} \mathcal{B}\left(\frac{1}{\alpha+1}, p-\frac{1}{\alpha+1}\right),
$$

where $\mathcal{B}$ is the Beta function and

$$
\sum_{|\nu|>N} \frac{1}{\left(1+|\nu h|^{\alpha+1}\right)^{p}} \leq \frac{2 h^{-(\alpha+1) p}}{((\alpha+1) p-1) N^{(\alpha+1) p-1}} .
$$

Combining (3.15), (3.14), and (3.13) yields

$$
\left(\sum_{k \in \mathbb{Z}_{\ell, N}^{n}}|f(k h)|^{p}\right)^{1 / p} \leq \frac{A 2^{1 / p} \mathcal{D}^{n-1}(\alpha)}{h^{\alpha+1}((\alpha+1) p-1)^{1 / p} N^{\alpha+1-1 / p}},
$$

where is defined in (3.11). Substituting (3.16) and (2.21) into (3.12) implies (3.10).

The decay of the uniform bound in (3.10) depends on the convergence factor $\varphi_{\ell}$, $\ell=1, \ldots, n$, and it is of order $O\left(\sum_{\ell=1}^{n} \alpha_{\ell}(N) / N^{\alpha+1-1 / p}\right)$ as $N \rightarrow \infty$. The decay of the uniform bound of the WKS sampling series (1.2) was investigated by Long and Fang in [20, Theorem 1] associated with the decay condition

$$
|f(x)| \leq \frac{A}{(1+\|x\|)^{n(1 / p+\delta)}},
$$

where $\delta>0$ and $\|x\|=\left(\sum_{j=1}^{n}\left|x_{j}\right|^{2}\right)^{1 / 2}$. The decay of the Long-Fang bound is of the order of $O\left(N^{-\delta}\right)$ as $N \rightarrow \infty$.

According to Nikol'skii [21], the decay of $B_{\sigma}^{p}\left(\mathbb{R}^{n}\right)$-functions is limited. It is known that the function in the space $B_{\sigma}^{p}\left(\mathbb{R}^{n}\right)$ cannot decay faster than

$$
|f(x)| \leq A \prod_{j=1}^{n} e^{-a_{j}\left|x_{j}\right|^{m_{j}}}
$$


where $\left.m_{j} \in\right] 0,1$, for all $j=1, \ldots, n$, and $a_{j}$ are positive numbers. In the following theorem we extend the work of Annaby and Asharabi in [1, Theorem 3.4] to functions from the space $B_{\sigma}^{p}\left(\mathbb{R}^{n}\right)$ having a decay of the form (3.17).

THEOREM 3.5. Let $f \in B_{(1-\theta) \sigma}^{p}\left(\mathbb{R}^{n}\right)$ satisfies the decay condition (3.17). Then we have the following uniform bound:

$$
\left|T_{N}(x)\right| \leq A p^{n} \sum_{\ell=1}^{n} \alpha_{\ell}(N)\left(\frac{\left(\left\lfloor 1 / m_{\ell}\right\rfloor+1\right) ! N}{m_{\ell}}\right)^{1 / p} e^{-a_{\ell}(N h)^{m_{\ell}}} \prod_{j=1, j \neq \ell}^{n} \phi_{j}\left\|\varphi_{j}\right\|_{\infty},
$$

where

$$
\phi_{j}=\left(1+\frac{2}{h m_{j}\left(p a_{j}\right)^{1 / m_{j}}} \Gamma\left(\frac{1}{m_{j}}\right)\right)^{1 / p},
$$

$\Gamma$ is the Gamma function, and $\lfloor 1 / m\rfloor$ is the greatest integer smaller than $1 / m$.

Proof. Since $f \in B_{(1-\theta) \sigma}^{p}\left(\mathbb{R}^{n}\right)$, the sampling series expansion (3.1) and inequality (3.12) hold. From condition (3.17), we have for a fixed $\ell$

$$
\sum_{k \in \mathbb{Z}_{\ell, N}^{n}}|f(k h)|^{p} \leq A^{p} \sum_{k \in \mathbb{Z}_{\ell, N}^{n}} \prod_{j=1}^{n} e^{-p a_{j}\left(k_{j} h\right)^{m_{j}}} .
$$

In [1, Lemma 2.5], we have

$$
\sum_{n>N}^{\infty} e^{-\beta|n h|^{m}}<\left(\frac{(\lfloor 1 / m\rfloor+1) ! N}{m}\right) e^{-\beta(N h)^{m}},
$$

where $m \in] 0,1[, \beta>0$, and $h N>1$. Under the same assumptions as for (3.21), the authors of [7] have established the estimate

$$
\sum_{n=-\infty}^{\infty} e^{-\beta|n h|^{m}}<1+\frac{2}{h m \beta^{1 / m}} \Gamma\left(\frac{1}{m}\right) .
$$

Substituting (3.22) and (3.21) into (3.20), we obtain for a fixed $\ell$ that

$$
\sum_{k \in \mathbb{Z}_{\ell, N}^{n}}|f(k h)|^{p} \leq A^{p}\left(\frac{\left(\left\lfloor 1 / m_{\ell}\right\rfloor+1\right) ! N}{m_{\ell}}\right) e^{-p a_{\ell}(N h)^{m_{\ell}}} \prod_{j=1, j \neq \ell}^{n} \phi_{j}^{p}\left(m_{j}\right) .
$$

Combining (3.23), (2.21), and (3.12) implies (3.18).

The decay of the above bound depends on the convergence factor $\varphi_{\ell}, \ell=1, \ldots, n$, and it is of order $O\left(\sum_{\ell=1}^{n} e^{-a_{\ell}(N h)^{m_{\ell}}} \alpha_{\ell}(N) N^{1 / p}\right)$ as $N \rightarrow \infty$.

Motivated by the work of Ye in [25], we truncate the series on the right-hand side of (3.1) based on localized sampling as follows:

$$
\mathcal{E}_{N}(x)=\sum_{k \in \mathbb{Z}_{N}^{n}(x)} f(k h) \prod_{j=1}^{n} \varphi_{j}\left(h^{-1} x_{j}-k_{j}\right) \operatorname{sinc}\left(\pi h^{-1} x_{j}-k_{j} \pi\right),
$$

where $N$ is any positive number, $x \in \mathbb{R}^{n}$, and $\mathbb{Z}_{N}^{n}(x)$ is defined in (2.8). In this case, the truncation error associated with the series in (3.1) will be defined as

$$
\begin{aligned}
\mathcal{T}_{N}(x) & :=f(x)-\mathcal{E}_{N}(x), \\
& =\sum_{k \notin \mathbb{Z}_{N}(x)} f(k h) \prod_{j=1}^{n} \varphi_{j}\left(h^{-1} x_{j}-k_{j}\right) \operatorname{sinc}\left(\pi h^{-1} x_{j}-k_{j} \pi\right),
\end{aligned}
$$


for all $x \in \mathbb{R}^{n}$.

The next theorem gives a bound for this type of truncation error.

THEOREM 3.6. Let $f \in B_{(1-\theta) \sigma}^{p}\left(\mathbb{R}^{n}\right)$. Then for $x \in \mathbb{R}^{n}$ and $N \in \mathbb{N}$, we have

$$
\left|\mathcal{T}_{N}(x)\right| \leq \frac{\mathcal{S}_{p, h} p^{n-1}\|f\|_{p}}{N^{1 / p}} \sum_{\ell=1}^{n} \prod_{j=1, j \neq \ell}^{n}\left\|\varphi_{j}\right\|_{\infty} \alpha_{\ell}(N),
$$

where $\mathcal{S}_{p, h}$ is a positive constant depending on $p$, $h$, and the function $\alpha_{\ell}$ is given in (2.10).

Proof. Since $f \in B_{(1-\theta) \sigma}^{p}\left(\mathbb{R}^{n}\right)$, the sampling series (3.1) holds. From (3.24), we obtain

$$
\left|\mathcal{T}_{N}(x)\right| \leq \sum_{k \notin \mathbb{Z}_{N}(x)}\left|f(k h) \prod_{j=1}^{n} \varphi_{j}\left(h^{-1} x_{j}-k_{j}\right) \operatorname{sinc}\left(\pi h^{-1} x_{j}-k_{j} \pi\right)\right| .
$$

Applying the generalized Hölder inequality implies

$$
\begin{aligned}
\left|\mathcal{T}_{N}(x)\right| \leq & \left(\sum_{k \notin \mathbb{Z}_{N}(x)}|f(k h)|^{p}\right)^{1 / p} \\
& \left(\sum_{k \notin \mathbb{Z}_{N}(x)}\left|\prod_{j=1}^{n} \varphi_{j}\left(h^{-1} x_{j}-k_{j}\right) \operatorname{sinc}\left(\pi h^{-1} x_{j}-k_{j} \pi\right)\right|^{q}\right)^{1 / q} .
\end{aligned}
$$

Since $f \in B_{(1-\theta) \sigma}^{p}\left(\mathbb{R}^{n}\right) \subset B_{\sigma}^{p}\left(\mathbb{R}^{n}\right)$, we have

$$
\left(\sum_{k \notin \mathbb{Z}_{N}(x)}|f(k h)|^{p}\right)^{1 / p} \leq\left(\sum_{k \in \mathbb{Z}^{n}}|f(k h)|^{p}\right)^{1 / p} \leq A_{p, h}\|f\|_{p},
$$

where $A_{p, h}$ is a positive constant depending on $p$ and $h$. The last inequality in (3.27) was already stated in the literature, cf., e.g., [21, pp. 123-124]. Combining (3.27), (3.26), and (2.9) implies (3.25).

The decay of the above bound depends on the convergence factor $\varphi_{\ell}, \ell=1, \ldots, n$, and is of order $O\left(\sum_{\ell=1}^{n} \alpha_{\ell}(N) / N^{1 / p}\right)$ as $N \rightarrow \infty$. Ye's bound, cf. [25, Theorem 1], for the truncation error of the series in (1.2) is of order $O\left(N^{-1 / p}\right)$ as $N \rightarrow \infty$.

4. Perturbation error bounds. In most approximation problems, the samples $f(k h)$, $k \in \mathbb{Z}^{n}$, are observations that are not exactly available. There are two famous approximations for the samples $f(k h)$. The first approximation $\tilde{f}(k h)$ is located at the original nodes, and the occuring error is called amplitude error. In the second approximation, the samples $f(k h)$ are not exactly available at the original nodes $\{k h\}_{k \in \mathbb{Z}^{n}}$ but at the nodes $\left\{k h+\delta_{k}\right\}_{k \in \mathbb{Z}^{n}}$, where $\delta_{k}:=\left(\delta_{k_{1}}, \delta_{k_{2}}, \ldots, \delta_{k_{n}}\right)$ and $\left\|\delta_{k}\right\|<\delta \ll 1$. This type of error is called jitter error. In this section, we establish bounds for these perturbation errors using certain techniques like those of Butzer and Splettstösser in [9] and Annaby and Asharabi in [4]. To the best of our knowledge, this is the first time that these types of errors are investigated for a multidimensional sampling series.

4.1. Amplitude error. The amplitude error arises if the exact sampling values $f(k h)$, $k \in \mathbb{Z}^{n}$, are replaced by approximate ones $\tilde{f}(k h)$ in the regularized multidimensional sampling 
series (3.1). The amplitude error is defined in this case to be

$$
A_{\varepsilon}[f](x):=\sum_{k \in \mathbb{Z}^{n}}\{f(k h)-\tilde{f}(k h)\} \prod_{j=1}^{n} \varphi_{j}\left(h^{-1} x_{j}-k_{j}\right) \operatorname{sinc}\left(\pi h^{-1} x_{j}-k_{j} \pi\right) .
$$

For $k \in \mathbb{Z}^{n}$, we assume that

$$
\varepsilon_{k}:=f(k h)-\tilde{f}(k h), \quad\left|\varepsilon_{k}\right|<\varepsilon .
$$

It is well known that the Bernstein functions satisfy decay conditions when $\left|x_{\ell}\right| \rightarrow \infty$, $1 \leq \ell \leq n$; see [21, p. 109]. Therefore, let us suppose that the differences $\varepsilon_{k}$ satisfies the condition

$$
\left|\varepsilon_{k}\right| \leq|f(k h)|, \quad k \in \mathbb{Z}^{n} .
$$

In the following theorem, we estimate the amplitude error when the function has a decay of the form (3.9).

THEOREM 4.1. Let $f \in B_{(1-\theta) \sigma}^{\infty}\left(\mathbb{R}^{n}\right)$ such that conditions (3.9) and (4.2) hold. Then, for $0<\varepsilon \leq \min \left\{h, h^{-1}, 1 / \sqrt{e}\right\}$, we have

$$
\left\|A_{\varepsilon}[f]\right\|_{\infty} \leq \frac{4^{n} \rho_{n}(\alpha)}{(\alpha+1)^{n}} \prod_{j=1}^{n}\left\|\varphi_{j}\right\|_{\infty} \varepsilon \log ^{n}(1 / \varepsilon)
$$

where

$$
\rho_{n}(\alpha):=3^{n(\alpha+1) / 2} e^{n}+n A \sqrt{2} e^{1 / 4} \mathcal{D}^{n-1}(\alpha)
$$

and the function $\mathcal{D}(\alpha)$ is defined in (3.11). Moreover, the right-hand side of (4.3) tends to zero as $\varepsilon \rightarrow 0$.

Proof. Since $f$ is in $B_{(1-\theta) \sigma}^{\infty}\left(\mathbb{R}^{n}\right)$ and satisfies the decay condition (3.9), it follows that $f \in B_{(1-\theta) \sigma}^{p}\left(\mathbb{R}^{n}\right)$ for all $p>1$ and the regularized expansion (3.1) holds. Applying the generalized Hölder inequality and using Lemma 2.1 for (4.1), we obtain

$$
\begin{aligned}
&\left|A_{\varepsilon}[f](x)\right| \leq\left(\sum_{k \in \mathbb{Z}^{n}}\left|\varepsilon_{k}\right|^{p}\right)^{1 / p}\left(\sum_{k \in \mathbb{Z}^{n}} \prod_{j=1}^{n}\left|\varphi_{j}\left(h^{-1} x_{j}-k_{j}\right) \operatorname{sinc}\left(\pi h^{-1} x_{j}-k_{j} \pi\right)\right|^{q}\right)^{1 / q} \\
& \text { (4.4) } \quad \leq p^{n} \prod_{j=1}^{n}\left\|\varphi_{j}\right\|_{\infty}\left(\sum_{k \in \mathbb{Z}^{n}}\left|\varepsilon_{k}\right|^{p}\right)^{1 / p}
\end{aligned}
$$

for all $x \in \mathbb{R}^{n}$. Define the sequences $a_{k}, b_{k}$ to be $a_{k}:=\varepsilon_{k}, k \in \mathbb{B}_{N}^{n}$, and $b_{k}:=\varepsilon_{k}$, $k \in \mathbb{Z}^{n} / \mathbb{B}_{N}^{n}$, where $\mathbb{B}_{N}^{n}$ is defined in (3.2). Therefore $a_{k}+b_{k}=\varepsilon_{k}$ for all $k \in \mathbb{Z}^{n}$. Applying the generalized Minkowski inequality implies

$$
\left(\sum_{k \in \mathbb{Z}^{n}}\left|\varepsilon_{k}\right|^{p}\right)^{1 / p} \leq\left(\sum_{k \in \mathbb{B}_{N}^{n}}\left|\varepsilon_{k}\right|^{p}\right)^{1 / p}+\left(\sum_{k \in \mathbb{Z}^{n} / \mathbb{B}_{N}^{n}}\left|\varepsilon_{k}\right|^{p}\right)^{1 / p} .
$$

As we have done in (3.4), we obtain

$$
\left(\sum_{k \in \mathbb{Z}^{n} / \mathbb{B}_{N}^{n}}\left|\varepsilon_{k}\right|^{p}\right)^{1 / p} \leq \sum_{\ell=1}^{n}\left(\sum_{k \in \mathbb{Z}_{\ell, N}^{n}}\left|\varepsilon_{k}\right|^{p}\right)^{1 / p}
$$


Therefore,

$$
\left(\sum_{k \in \mathbb{Z}^{n}}\left|\varepsilon_{k}\right|^{p}\right)^{1 / p} \leq\left(\sum_{k \in \mathbb{B}_{N}^{n}}\left|\varepsilon_{k}\right|^{p}\right)^{1 / p}+\sum_{\ell=1}^{n}\left(\sum_{k \in \mathbb{Z}_{\ell, N}^{n}}\left|\varepsilon_{k}\right|^{p}\right)^{1 / p}
$$

Using (4.2), (3.9), and (3.16), we obtain for $N \geq\left\lfloor\frac{1}{h}\right\rfloor$,

$$
\begin{aligned}
\sum_{\ell=1}^{n}\left(\sum_{k \in \mathbb{Z}_{\ell, N}^{n}}\left|\varepsilon_{k}\right|^{p}\right)^{1 / p} & \leq \sum_{\ell=1}^{n}\left(\sum_{k \in \mathbb{Z}_{\ell, N}^{n}}|f(k h)|^{p}\right)^{1 / p} \\
& \leq \frac{n A 2^{1 / p} \mathcal{D}^{n-1}(\alpha)}{h^{\alpha+1}((\alpha+1) p-1)^{1 / p} N^{\alpha+1-1 / p}}
\end{aligned}
$$

where $N \geq 1$ and $\mathcal{D}(\alpha)$ is defined in (3.11). Moreover,

$$
\left(\sum_{k \in \mathbb{B}_{N}^{n}}\left|\varepsilon_{k}\right|^{p}\right)^{1 / p} \leq \varepsilon(2 N+1)^{n / p}
$$

Combining (4.4)-(4.7), we obtain

$$
\left\|A_{\varepsilon}[f]\right\|_{\infty} \leq p^{n} \prod_{j=1}^{n}\left\|\varphi_{j}\right\|_{\infty}\left\{\varepsilon(2 N+1)^{n / p}+\frac{n A 2^{1 / p} \mathcal{D}^{n-1}(\alpha)}{h^{\alpha+1}((\alpha+1) p-1)^{1 / p} N^{\alpha+1-1 / p}}\right\} .
$$

The parameters $N$ and $p$ in (4.8) can to be chosen suitably to obtain the desired estimate. Therefore, we choose $N$ and $p$ to be

$$
N:= \begin{cases}\left\lfloor\varepsilon^{-1 /(\alpha+1)} h^{\frac{-(\alpha+1) p}{(\alpha+1) p-1}}\right\rfloor, & h \leq 1, \\ \left\lfloor\varepsilon^{-1 /(\alpha+1)} h^{\frac{(\alpha+1) p}{(\alpha+1) p-1}}\right\rfloor, & h>1,\end{cases}
$$

and

$$
p=\frac{4}{\alpha+1} \log (1 / \varepsilon)
$$

Using the assumption $0<\varepsilon \leq \min \left\{h, h^{-1}, 1 / \sqrt{e}\right\}$ and (4.9), (4.10), we can find the following estimations, cf. [1, p. 353]:

$$
(2 N+1)^{1 / p} \leq 3^{(\alpha+1) / 2} e, \quad h^{-\alpha-1} N^{\frac{1-(\alpha+1) p}{p}} \leq e^{1 / 4} \varepsilon .
$$

Since $0<\varepsilon \leq \min \left\{h, h^{-1}, 1 / \sqrt{e}\right\}$ and (4.10), we have $(\alpha+1) p \geq 2$ and therefore $1 /((\alpha+1) p-1)^{1 / p} \geq 1$. Combining (4.11) and (4.8) implies (4.3). Since $\varepsilon \log ^{n}(1 / \varepsilon) \rightarrow 0$ as $\varepsilon \rightarrow 0$, the right-hand side of (4.3) tends to zero as $\varepsilon \rightarrow 0$.

In the next theorem, we establish another bound for the amplitude error for a function that satisfies a decay condition of the form (3.17).

THEOREM 4.2. Let $f \in B_{(1-\theta) \sigma}^{\infty}\left(\mathbb{R}^{n}\right)$ such that conditions (3.17) and (4.2) hold. Then, for $0<\varepsilon \leq \min \left\{h, h^{-1}, 1 / \sqrt{e}\right\}$, we have

$$
\left\|A_{\varepsilon}[f]\right\|_{\infty} \leq 4^{n} \varrho_{n} \prod_{j=1}^{n}\left\|\varphi_{j}\right\|_{\infty} \varepsilon \log ^{n}(1 / \varepsilon)
$$


where

$$
\varrho_{n}:=3^{n / 2} e^{n}+A e \sum_{\ell=1}^{n}\left(\frac{\left(\left\lfloor 1 / m_{\ell}\right\rfloor+1\right) !}{m_{\ell}}\right)^{1 / 2} \frac{\nu_{\ell} !}{a_{\ell}^{\nu_{\ell}}} \prod_{k=1, k \neq \ell}^{n} \phi_{k}\left(m_{k}\right),
$$

$\left.m_{\ell} \in\right] 0,1\left[,\left\lfloor m_{\ell} \nu_{\ell}\right\rfloor=1\right.$, and $\phi_{k}$ is defined in (3.19). Moreover, the right-hand side of (4.12) tends to zero as $\varepsilon \rightarrow 0$.

Proof. Since $f$ is in $B_{(1-\theta) \sigma}^{\infty}\left(\mathbb{R}^{n}\right)$ and satisfies the decay condition (3.17), it follows that $f \in B_{(1-\theta) \sigma}^{p}\left(\mathbb{R}^{n}\right)$ for all $p>1$ and the regularized expansion (3.1) holds. Therefore, the inequalities (4.4) and (4.5) are also satisfied. Using conditions (4.2) and (3.17), we obtain for $1 \leq \ell \leq n$ and $N h>1$,

$$
\begin{aligned}
\left(\sum_{k \in \mathbb{Z}_{\ell, N}^{n}}\left|\varepsilon_{k}\right|^{p}\right)^{1 / p} & \leq\left(\sum_{k \in \mathbb{Z}_{\ell, N}^{n}}|f(k h)|^{p}\right)^{1 / p} \\
& \leq A\left(\frac{\left(\left\lfloor 1 / m_{\ell}\right\rfloor+1\right) ! N}{m_{\ell}}\right)^{1 / p} e^{-a_{\ell}(N h)^{m_{\ell}}} \prod_{k=1, k \neq \ell}^{n} \phi_{k}\left(m_{k}\right),
\end{aligned}
$$

where we have used (3.23) in the last step of inequality (4.13). Combining (4.7), (4.13), (4.4), and (4.5) yields

$$
\begin{aligned}
\left|A_{\varepsilon}[f](x)\right| \leq & p^{n} \prod_{j=1}^{n}\left\|\varphi_{j}\right\|_{\infty}\left\{\varepsilon(2 N+1)^{n / p}\right. \\
& \left.+A \sum_{\ell=1}^{n}\left(\frac{\left(\left\lfloor 1 / m_{\ell}\right\rfloor+1\right) ! N}{m_{\ell}}\right)^{1 / p} e^{-a_{\ell}(N h)^{m_{\ell}}} \prod_{k=1, k \neq \ell}^{n} \phi_{k}\left(m_{k}\right)\right\} .
\end{aligned}
$$

As we have done in the proof of the previous theorem, we will choose the parameters $p$ and $N$ appropriately to obtain the desired bound. Let $p$ and $N$ be

$$
p=4 \log (1 / \varepsilon), \quad N:= \begin{cases}\left\lfloor\frac{h}{\varepsilon}\right\rfloor, & h \leq 1, \\ \left\lfloor\frac{1}{h \varepsilon}\right\rfloor, & h>1 .\end{cases}
$$

From the assumption $0<\varepsilon \leq \min \left\{h, h^{-1}, 1 / \sqrt{e}\right\}$ and (4.15), we have, cf. [1, p. 355],

$$
(2 N+1)^{1 / p} \leq \sqrt{3} e, \quad N^{\frac{1}{p}} \leq e, \quad e^{-a_{\ell}(N h)^{m_{\ell}}} \leq \frac{\nu_{\ell} ! \varepsilon}{a_{\ell}^{\nu_{\ell}}}
$$

where $\left\lfloor m_{\ell} \nu_{\ell}\right\rfloor=1$. Combining (4.16) and (4.14) and noting that $p \geq 2$, we arrive at (4.12). Clearly, the right-hand side of (4.12) tends to zero as $\varepsilon \rightarrow 0$.

4.2. Jitter error. In this section, we investigate bounds for the jitter error. We will use the same decay conditions as in the previous section. In addition, we present a bound for the jitter error without any decay condition. The jitter error appears when rebuilding an approximate function in $f \in B_{\sigma}^{p}\left(\mathbb{R}^{n}\right), p>1$, from samples taken at nodes nearby the exact original ones. In other words, let $\delta_{k}:=\left(\delta_{k_{1}}, \ldots, \delta_{k_{n}}\right) \in \mathbb{R}^{n}$ denote a set of perturbation 
values. Then the jitter error $J_{\delta}$ associated with the regularized multidimensional sampling series (3.1) is defined by

$$
J_{\delta}[f](x):=\sum_{k \in \mathbb{Z}^{n}}\left\{f(k h)-f\left(k h+\delta_{k}\right)\right\} \prod_{j=1}^{n} \varphi_{j}\left(h^{-1} x_{j}-k_{j}\right) \operatorname{sinc}\left(\pi h^{-1} x_{j}-k_{j} \pi\right),
$$

where $x \in \mathbb{R}^{n}$ and $\left\|\delta_{k}\right\|_{\mathbb{R}^{n}} \leq \delta$, for all $k \in \mathbb{Z}^{n}$.

THEOREM 4.3. Let $f \in B_{(1-\theta) \sigma}^{\infty}\left(\mathbb{R}^{n}\right)$ such that condition (3.9) holds. Then, for $0<\delta \leq \min \left\{h, h^{-1}, 1 / \sqrt{e}\right\}$, we have

$$
\left\|J_{\delta}[f]\right\|_{\infty} \leq \frac{4^{n} \omega_{n}(\alpha)}{(\alpha+1)^{n}} \prod_{j=1}^{n}\left\|\varphi_{j}\right\|_{\infty} \delta \log ^{n}(1 / \delta),
$$

where

$$
\omega_{n}(\alpha):=3^{n(\alpha+1) / 2} e^{n} \sum_{\ell=1}^{n}\left\|f_{x_{\ell}}^{\prime}\right\|_{\infty}+n A 2^{3 / 2} e^{1 / 4} \mathcal{D}^{n-1}(\alpha)
$$

and the function $\mathcal{D}(\alpha)$ is defined in (3.11). Furthermore, the right-hand side of (4.18) tends to zero as $\delta \rightarrow 0$.

Proof. Since $f \in B_{(1-\theta) \sigma}^{\infty}\left(\mathbb{R}^{n}\right)$ satisfies the decay condition (3.9), $f \in B_{(1-\theta) \sigma}^{p}\left(\mathbb{R}^{n}\right)$ for all $p>1$, and the regularized expansion (3.1) holds. Applying the generalized Hölder inequality to (4.17) and using Lemma 2.1, we obtain for $x \in \mathbb{R}^{n}$,

$$
\begin{aligned}
\left|J_{\delta}[f](x)\right| \leq\left(\sum_{k \in \mathbb{Z}^{n}}\left|f(k h)-f\left(k h+\delta_{k}\right)\right|^{p}\right)^{1 / p} \\
\quad \times\left(\sum_{k \in \mathbb{Z}^{n}} \prod_{j=1}^{n}\left|\varphi_{j}\left(h^{-1} x_{j}-k_{j}\right) \operatorname{sinc}\left(\pi h^{-1} x_{j}-k_{j} \pi\right)\right|^{q}\right)^{1 / q} \\
\leq p^{n} \prod_{j=1}^{n}\left\|\varphi_{j}\right\|_{\infty}\left(\sum_{k \in \mathbb{Z}^{n}}\left|f(k h)-f\left(k h+\delta_{k}\right)\right|^{p}\right)^{1 / p} .
\end{aligned}
$$

Applying the generalized Minkowski inequality and using the same technique of (4.5), we get

$$
\begin{aligned}
\left(\sum_{k \in \mathbb{Z}^{n}}\left|f(k h)-f\left(k h+\delta_{k}\right)\right|^{p}\right)^{1 / p} & \\
\leq & \left(\sum_{k \in \mathbb{B}_{N}^{n}}\left|f(k h)-f\left(k h+\delta_{k}\right)\right|^{p}\right)^{1 / p} \\
& +\sum_{\ell=1}^{n}\left(\sum_{k \in \mathbb{Z}_{\ell, N}^{n}}\left|f(k h)-f\left(k+\delta_{k}\right)\right|^{p}\right)^{1 / p},
\end{aligned}
$$


where $\mathbb{B}_{N}^{n}$ is defined in (3.2). Applying the generalized Minkowski inequality and using condition (3.9) and inequality (3.16), we have

$$
\left(\sum_{k \in \mathbb{Z}_{\ell, N}^{n}}\left|f(k h)-f\left(k h+\delta_{k}\right)\right|^{p}\right)^{1 / p} \leq \frac{2 A 2^{1 / p} \mathcal{D}^{n-1}(\alpha)}{h^{\alpha+1}((\alpha+1) p-1)^{1 / p} N^{\alpha+1-1 / p}} .
$$

From the mean value theorem for multivariate functions, cf., e.g., [12, Theorem 3.4], we have

$$
f(k h)-f\left(k h+\delta_{k}\right)=\sum_{\ell=1}^{n} f_{x_{\ell}}^{\prime}(a) \delta_{k_{\ell}}
$$

where $f_{x_{\ell}}^{\prime}:=\partial f / \partial x_{\ell}$ and $a=\left(a_{1}, \ldots, a_{n}\right)$ is an interior point in the line sequent $L$ between the points $k h$ and $k h+\delta_{k}$. Therefore, by applying the generalized Minkowski inequality, we obtain

$$
\begin{aligned}
\left(\sum_{k \in \mathbb{B}_{N}^{n}}\left|f(k h)-f\left(k h+\delta_{k}\right)\right|^{p}\right)^{1 / p} & \leq \sum_{\ell=1}^{n}\left(\sum_{k \in \mathbb{B}_{N}^{n}}\left|f_{x_{\ell}}^{\prime}(a) \delta_{k_{\ell}}\right|^{p}\right)^{1 / p} \\
& \leq \delta(2 N+1)^{n / p} \sum_{\ell=1}^{n}\left\|f_{x_{\ell}}^{\prime}\right\|_{\infty},
\end{aligned}
$$

where we have used the fact that $\left|\delta_{k_{\ell}}\right| \leq\left\|\delta_{k}\right\|_{\mathbb{R}^{n}} \leq \delta$, for all $\ell=1, \ldots, n$, and that $\left\|f_{x_{\ell}}^{\prime}\right\|_{\infty}=\sup _{a \in L}\left|f_{x_{\ell}}^{\prime}(a)\right|$. The set $\mathbb{B}_{N}^{n}$ is defined in (3.2). Combining (4.19), (4.20), (4.21), and (4.22), we obtain

$$
\begin{aligned}
\left|J_{\delta}[f](x)\right| \leq p^{n} \prod_{j=1}^{n}\left\|\varphi_{j}\right\|_{\infty}\{\delta & (2 N+1)^{n / p} \sum_{\ell=1}^{n}\left\|f_{x_{\ell}}^{\prime}\right\|_{\infty} \\
& \left.+\frac{n A 2^{1+1 / p} \mathcal{D}^{n-1}(\alpha)}{h^{\alpha+1}((\alpha+1) p-1)^{1 / p} N^{\alpha+1-1 / p}}\right\} .
\end{aligned}
$$

As we have done before, we choose $N$ and $p$ to be

$$
N:= \begin{cases}\left\lfloor\delta^{-1 /(\alpha+1)} h^{\left.\frac{-(\alpha+1) p}{(\alpha+1) p-1}\right\rfloor,}, \quad h \leq 1,\right. \\ \left\lfloor\delta^{-1 /(\alpha+1)} h^{\left.\frac{(\alpha+1) p}{(\alpha+1) p-1}\right\rfloor,}, \quad h>1\right.\end{cases}
$$

and

$$
p=\frac{4}{\alpha+1} \log (1 / \delta)
$$

Using the assumption $0<\delta \leq \min \left\{h, h^{-1}, 1 / \sqrt{e}\right\}$ and (4.24), (4.25), we obtain

$$
(2 N+1)^{1 / p} \leq 3^{(\alpha+1) / 2} e, \quad h^{-\alpha-1} N^{\frac{1-(\alpha+1) p}{p}} \leq e^{1 / 4} \delta .
$$

Since $0<\delta \leq \min \left\{h, h^{-1}, 1 / \sqrt{e}\right\}$ and by (4.10), we have $(\alpha+1) p \geq 2$ and therefore $1 /((\alpha+1) p-1)^{1 / p} \geq 1$. Substituting (4.26) into (4.23) yields (4.18). It is easy to show that the right-hand side of (4.18) tends to zero as $\delta \rightarrow 0$. 
The next theorem investigates other bounds for the jitter error when the function $f$ satisfies decay conditions of the form (3.17).

THEOREM 4.4. Let $f \in B_{(1-\theta) \sigma}^{\infty}\left(\mathbb{R}^{n}\right)$ such that conditions (3.17) and (4.2) hold. Then, for $0<\delta \leq \min \left\{h, h^{-1}, 1 / \sqrt{e}\right\}$, we have

$$
\left\|J_{\delta}[f]\right\|_{\infty} \leq 4^{n} \mu_{n} \prod_{j=1}^{n}\left\|\varphi_{j}\right\|_{\infty} \delta \log ^{n}(1 / \delta)
$$

where

$$
\mu_{n}:=\sum_{\ell=1}^{n}\left\{3^{n / 2} e^{n}\left\|f_{x_{\ell}}^{\prime}\right\|_{\infty}+A e \frac{\nu_{\ell} !}{a_{\ell}^{\nu_{\ell}}} \sqrt{\frac{\left(\left\lfloor 1 / m_{\ell}\right\rfloor+1\right) !}{m_{\ell}}} \prod_{k=1, k \neq \ell}^{n} \phi_{k}\left(m_{k}\right)\right\}
$$

and $\phi_{j}$ is defined in (3.19). Furthermore, the right-hand side of (3.19) tends to zero when $\delta \rightarrow 0$.

Proof. Since $f$ is in $B_{(1-\theta) \sigma}^{\infty}\left(\mathbb{R}^{n}\right)$ and satisfies the decay condition (3.17), it follows that $f \in B_{(1-\theta) \sigma}^{p}\left(\mathbb{R}^{n}\right)$ for all $p>1$ and the regularized expansion (3.1) holds. Therefore, inequality (4.19) is also satisfied. Applying the generalized Minkowski inequality and using (3.17), we obtain

$$
\begin{aligned}
\left(\sum_{k \in \mathbb{Z}_{\ell, N}^{n}} \mid f(k h)-\right. & \left.\left.f\left(k h+\delta_{k}\right)\right|^{p}\right)^{1 / p} \\
& \leq 2 A\left(\frac{\left(\left\lfloor 1 / m_{\ell}\right\rfloor+1\right) ! N}{m_{\ell}}\right)^{1 / p} e^{-a_{\ell}(N h)^{m_{\ell}}} \prod_{j=1, j \neq \ell}^{n} \phi_{j}\left(m_{j}\right),
\end{aligned}
$$

where we have used (3.23) in the last step of inequality (4.28). Combining (4.22) and (4.28) implies

$$
\begin{aligned}
& \left|J_{\delta}[f](x)\right| \\
& \quad \leq p^{n} \prod_{j=1}^{n}\left\|\varphi_{j}\right\|_{\infty}\left\{\delta(2 N+1)^{n / p} \sum_{\ell=1}^{n}\left\|f_{x_{\ell}}^{\prime}\right\|_{\infty}\right.
\end{aligned}
$$

$$
\left.+2 A \sum_{\ell=1}^{n}\left(\frac{\left(\left\lfloor 1 / m_{\ell}\right\rfloor+1\right) ! N}{m_{\ell}}\right)^{1 / p} e^{-a_{\ell}(N h)^{m_{\ell}}} \prod_{j=1, j \neq \ell}^{n} \phi_{j}\left(m_{j}\right)\right\} .
$$

Now, we choose $p$ and $N$ as

$$
p=4 \log (1 / \delta), \quad N:= \begin{cases}\left\lfloor\frac{h}{\delta}\right\rfloor, & h \leq 1, \\ \left\lfloor\frac{1}{h \delta}\right\rfloor, & h>1 .\end{cases}
$$

From the assumption $0<\delta \leq \min \left\{h, h^{-1}, 1 / \sqrt{e}\right\}$ and (4.30), we have

$$
(2 N+1)^{1 / p} \leq \sqrt{3} e, \quad N^{\frac{1}{p}} \leq e, \quad e^{-a_{\ell}(N h)^{m_{\ell}}} \leq \frac{\nu_{\ell} ! \delta}{a_{\ell}^{\nu_{\ell}}},
$$


where $\left\lfloor m_{\ell} \nu_{\ell}\right\rfloor=1$. Combining (4.29) and (4.31) and noting that $p \geq 2$, we obtain (4.27). It is obvious that the right-hand side of (4.27) tends to zero as $\delta \rightarrow 0$.

In Theorems 4.3 and 4.4, we used different decay conditions, and we get bounds of order $O\left(\delta \log ^{n}(1 / \delta)\right)$. In the following theorem, we will use similar technique as in [1, Theorem 4.7] and [3, Theorem 5.3] to establish a bound for the jitter error without any decay condition. This bound will be of order $O\left(\delta^{1 / 2}\right)$. Before that, we introduce the following lemma:

LEMMA 4.5. Let $f \in B_{\sigma}^{p}\left(\mathbb{R}^{n}\right), 1 \leq p<\infty$, then we have

$$
\sum_{k_{n}=-\infty}^{\infty} \ldots \sum_{k_{2}=-\infty}^{\infty} \int_{-\infty}^{\infty}\left|f\left(x_{1}, k_{2} h, \ldots, k_{n} h\right)\right|^{2} d x_{1}=h\|f\|_{2}^{2} .
$$

Proof. Since $f \in B_{\sigma}^{p}\left(\mathbb{R}^{n}\right), f\left(x_{1}, k_{2} h, \ldots, k_{n} h\right) \in B_{\sigma}^{p}(\mathbb{R})$, and we have the following one-dimensional WKS expansion:

$$
f\left(x_{1}, k_{2} h, \ldots, k_{n} h\right)=\sum_{k_{1}=-\infty}^{\infty} f\left(k_{1} h, k_{2} h, \ldots, k_{n} h\right) \operatorname{sinc}\left(\pi h^{-1} x_{1}-k_{1} \pi\right) .
$$

Applying the univariate Parseval's equality to (4.33) yields

$$
\int_{-\infty}^{\infty}\left|f\left(x_{1}, k_{2} h, \ldots, k_{n} h\right)\right|^{2} d x_{1}=h \sum_{k_{1}=-\infty}^{\infty}\left|f\left(k_{1} h, k_{2} h, \ldots, k_{n} h\right)\right|^{2} .
$$

Therefore, we have

$$
\sum_{k_{n}=-\infty}^{\infty} \ldots \sum_{k_{2}=-\infty}^{\infty} \int_{-\infty}^{\infty}\left|f\left(x_{1}, k_{2} h, \ldots, k_{n} h\right)\right|^{2} d x_{1}=h \sum_{k \in \mathbb{Z}^{n}}|f(k h)|^{2}=\|f\|_{2}^{2} / h^{n-1}
$$

where we have applied the multivariate Parseval's equality, cf., e.g., [16, p.158], in the last step.

THEOREM 4.6. Let $f \in B_{(1-\theta) \sigma}^{2}\left(\mathbb{R}^{n}\right)$. Then, for sufficiently small $\delta$, we have

$$
\left\|J_{\delta}[f]\right\|_{\infty} \leq 2^{n} \sqrt{\delta / h^{n-1}} \prod_{k=1}^{n}\left\|\varphi_{k}\right\|_{\infty} \sum_{\ell=1}^{n}\left\|f_{x_{\ell}}^{\prime}\right\|_{2}
$$

Proof. Since $f \in B_{(1-\theta) \sigma}^{2}\left(\mathbb{R}^{n}\right)$, the regularized expansion (3.1) holds. Therefore, we can use the inequality (4.17) with $p=q=2$. It is not difficult to verify that

$$
f\left(k h+\delta_{k}\right)-f(k h)=\sum_{\ell=1}^{n} \int_{k_{\ell} h}^{k_{\ell} h+\delta_{k_{\ell}}} f_{x_{\ell}}^{\prime}\left(x^{\ell}\right) d x_{\ell}
$$

where $x^{\ell}:=\left(x_{1}, x_{2}, \ldots, x_{n}\right)$ such that

$$
x_{k_{j}}:= \begin{cases}k_{j} h, & j>\ell \\ x_{k_{\ell}}, & j=\ell \\ k_{j} h+\delta_{j}, & j<\ell\end{cases}
$$

for all $\ell=1, \ldots, n$. Using (4.35) and applying the generalized Cauchy-Schwartz inequality for sums, we obtain

$$
\left(\sum_{k \in \mathbb{Z}^{n}}\left|f(k h)-f\left(k h+\delta_{k}\right)\right|^{2}\right)^{1 / 2} \leq \sum_{\ell=1}^{n}\left(\sum_{k \in \mathbb{Z}^{n}}\left|\int_{k_{\ell} h}^{k_{\ell} h+\delta_{k_{\ell}}} f_{x_{\ell}}^{\prime}\left(x^{\ell}\right) d x_{\ell}\right|^{2}\right)^{1 / 2}
$$


Applying the Cauchy-Schwartz inequality and noting that $\left|\delta_{k_{\ell}}\right| \leq \delta$ yields

$$
\begin{aligned}
\int_{k_{\ell} h}^{k_{\ell} h+\delta_{k_{\ell}}} f_{x_{\ell}}\left(x^{\ell}\right) d x_{\ell} & \leq\left(\int_{k_{\ell} h}^{k_{\ell} h+\delta_{k_{\ell}}} 1 d x_{\ell}\right)^{1 / 2}\left(\int_{k_{\ell} h}^{k_{\ell} h+\delta_{k_{\ell}}}\left|f_{x_{\ell}}^{\prime}\left(x^{\ell}\right)\right|^{2} d x_{\ell}\right)^{1 / 2} \\
& \leq \sqrt{\delta}\left(\int_{k_{\ell} h}^{k_{\ell} h+\delta_{k_{\ell}}}\left|f_{x_{\ell}}^{\prime}\left(x^{\ell}\right)\right|^{2} d x_{\ell}\right)^{1 / 2} .
\end{aligned}
$$

Combining (4.37), (4.36) and using (4.32) implies

$$
\begin{aligned}
\left(\sum_{k \in \mathbb{Z}^{n}}\left|f(k h)-f\left(k h+\delta_{k}\right)\right|^{2}\right)^{1 / 2} & \leq \sqrt{\delta} \sum_{\ell=1}^{n}\left(\sum_{k \in \mathbb{Z}^{n}} \int_{k_{\ell} h-\delta_{k_{\ell}}}^{k_{\ell} h+\delta_{k_{\ell}}}\left|f_{x_{\ell}}^{\prime}\left(x^{\ell}\right)\right|^{2} d x_{\ell}\right)^{1 / 2} \\
& \leq \sqrt{\delta} \sum_{\ell=1}^{n}\left(\sum_{k_{j} \in \mathbb{Z}, \forall j \neq \ell} \int_{-\infty}^{\infty}\left|f_{x_{\ell}}^{\prime}\left(x^{\ell}\right)\right|^{2} d x_{\ell}\right)^{1 / 2} \\
& =\sqrt{\delta / h^{n-1}} \sum_{\ell=1}^{n}\left\|f_{x_{\ell}}^{\prime}\right\|_{2},
\end{aligned}
$$

where $h \geq 2 \delta_{k_{\ell}}$ for all $\ell=1, \ldots, n$. Substituting (4.38) into (4.19) with $p=q=2$, we get (4.34).

5. Numerical illustrations. In this section, we introduce three numerical illustrations with a suitable choice for the functions $\varphi_{j}, j=1, \ldots, n$. The fastest decaying function in the Bernstein space is written as an infinite product of sinc-functions ([14]) so we can not use it here because it is not explicitly computable. Therefore, we choose $\varphi_{j}$ as the following finite product of sinc-functions

$$
\varphi_{j}\left(h^{-1} x_{j}-k_{j}\right):=\operatorname{sinc}^{m}\left((\theta \pi / m)\left(h^{-1} x_{j}-k_{j}\right)\right), \quad j=1, \ldots, n,
$$

where $0<\theta<1$. Obviously, $\prod_{j=1}^{n} \varphi_{j}\left(h^{-1} x_{j}-k_{j}\right) \in B_{\theta \sigma}^{p}\left(\mathbb{R}^{n}\right)$, and it satisfies the assumptions of Theorem 3.1. To give the reader a good illustration about the expansion (3.1), we provide graphs for the two examples. Furthermore, we compare our results with the results obtained from the WKS expansion (1.2). This comparison shows that the results of the regularized expansion (3.1) are more accurate than the results of the WKS expansion (1.2). Let $\mathfrak{T}_{N}$ be the truncation of the WKS series (1.2), i.e.,

$$
\mathfrak{T}_{N}(x):=f(x)-\sum_{k \in \mathbb{B}_{N}^{n}} f(k h) \prod_{j=1}^{n} \operatorname{sinc}\left(\pi h^{-1} x_{j}-k_{j} \pi\right),
$$

where $\mathbb{B}_{N}^{n}$ is defined in (3.2). We would like to point out that the comparison in the following three examples was done using the same samples $f(k h)$. The examples show that the accuracy of the approximation using the regularized expansion (3.1) decreases when we move away from the center of the approximation region. This phenomenon does not appear with the WKS expansion (1.2). The parameters $m$ and $\theta$ will be chosen as $m=\lfloor N / 2\rfloor$ and $\theta=\sigma / m$.

EXAMPLE 5.1. The function $f(t)=\frac{\sin (\pi t)}{t^{2}-1}, t \in \mathbb{R}$, belongs to $B_{\pi}^{p}(\mathbb{R})$. Therefore, the two expansions (1.2) and (3.1) hold with $n=1$. In Table 5.1 and Figures 5.1 and 5.2, we compare the approximations of the function $f$ at the points $t_{\ell}:=(\ell+1 / 2) h, \ell=0, \ldots, 5$, using the regularized and the WKS expansions with $N=20, m=10, \theta=\pi / 10$, and $h=1 / 2$. 
TABLE 5.1

Approximation of the function at $t_{\ell}$.

\begin{tabular}{ccc}
$t_{\ell}$ & \multicolumn{2}{c}{ Absolute error for the expansions $(1.2)$ and (3.1) } \\
\cline { 2 - 3 } & $\left|\mathfrak{T}_{20}\left(t_{\ell}\right)\right|$ & $\left|T_{20}\left(t_{\ell}\right)\right|$ \\
\hline$t_{0}$ & 0.000158485 & $5.29759 \times 10^{-8}$ \\
$t_{1}$ & 0.000159265 & $1.05962 \times 10^{-7}$ \\
$t_{2}$ & 0.000160848 & $2.43812 \times 10^{-7}$ \\
$t_{3}$ & 0.000163281 & $5.39095 \times 10^{-7}$ \\
$t_{4}$ & 0.000166641 & $1.12245 \times 10^{-6}$ \\
$t_{5}$ & 0.000171039 & $2.20790 \times 10^{-6}$
\end{tabular}

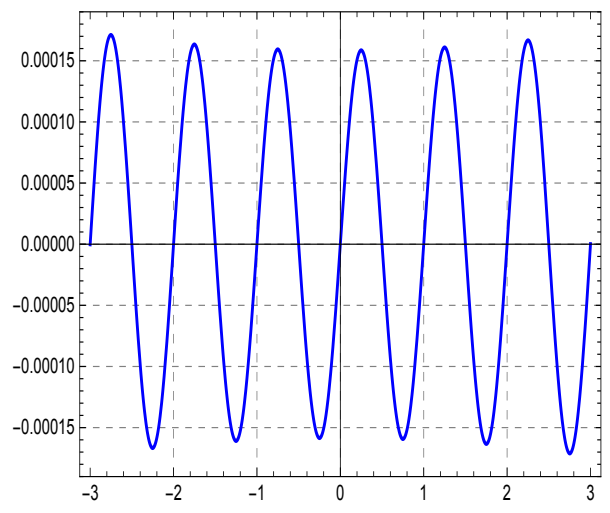

FIG. 5.1. $\mathfrak{T}_{20}(t), h=0.5$.

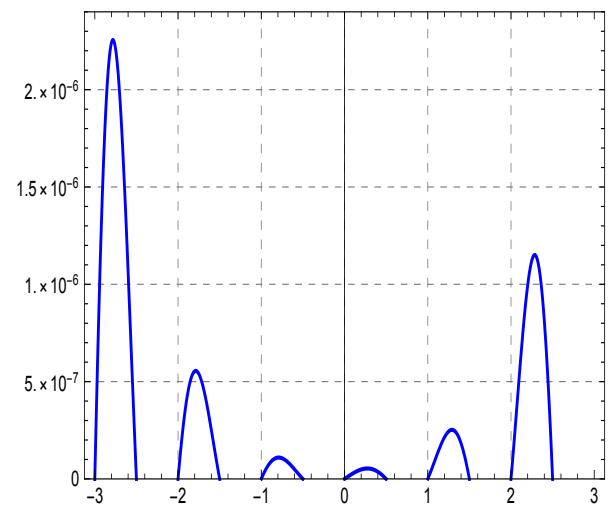

FIG. 5.2. $T_{20}(t), h=0.5, m=10, \theta=\pi / 10$.

EXAMPLE 5.2. Consider the function $f(x, y)=\operatorname{sinc}(\pi(x+y)) \in B_{\pi}^{p}\left(\mathbb{R}^{2}\right)$. Therefore, the two expansions (1.2) and (3.1) hold with $n=2$. In Table 5.2 and Figures 5.3 and 5.4, we summarize the comparison between the approximations of the function $f$ at the points $\left(x_{i}, y_{j}\right):=((i+1 / 2) h,(j+1 / 2) h), i=j=0, \ldots, 5$, using the regularized and the WKS expansions with $N=16, m=8, \theta=\pi / 8$, and $h=1 / 2$.

TABLE 5.2

Approximation of the function at $\left(x_{i}, y_{j}\right):=((i+1 / 2) h,(j+1 / 2) h)$.

\begin{tabular}{ccc}
$\left(x_{i}, y_{j}\right)$ & \multicolumn{2}{c}{ Absolute error for the expansions $(1.2)$ and (3.1) } \\
\cline { 2 - 3 } & $\left|\mathfrak{T}_{16}\left(x_{i}, y_{j}\right)\right|$ & $\left|T_{16}\left(x_{i}, y_{j}\right)\right|$ \\
\hline$\left(x_{0}, y_{0}\right)$ & 0.00121182 & $5.59929 \times 10^{-9}$ \\
$\left(x_{1}, y_{1}\right)$ & 0.00075818 & $3.88037 \times 10^{-8}$ \\
$\left(x_{2}, y_{2}\right)$ & 0.00076861 & $2.73004 \times 10^{-7}$ \\
$\left(x_{3}, y_{3}\right)$ & 0.00126320 & $7.35242 \times 10^{-7}$ \\
$\left(x_{4}, y_{4}\right)$ & 0.00129992 & $3.99209 \times 10^{-6}$ \\
$\left(x_{5}, y_{5}\right)$ & 0.00083769 & $5.93928 \times 10^{-6}$
\end{tabular}

EXAMPLE 5.3. Let $f(x, y, z)=\operatorname{sinc}\left(\pi \sqrt{1+x^{2}+y^{2}+z^{2}}\right)$. Obviously, $f \in B_{\pi}^{p}\left(\mathbb{R}^{3}\right)$, and the expansions (1.2) and (3.1) hold with $n=3$. In Table 5.3, we approximate $f$ at some 


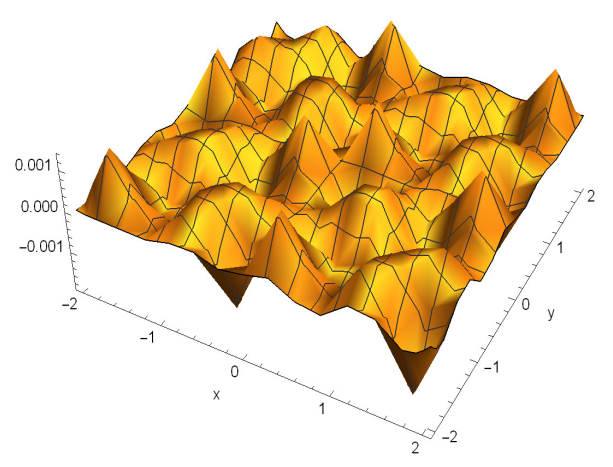

FIG. 5.3. $\mathfrak{T}_{16}(x, y), h=0.5$.

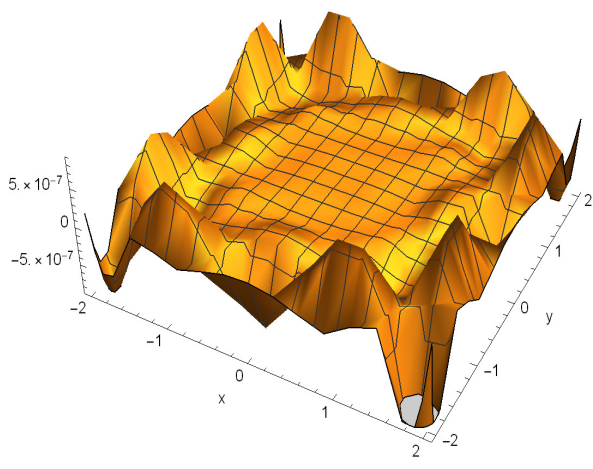

FIG. 5.4. $T_{16}(x, y), h=0.5, m=8, \theta=\pi / 8$.

points using the regularized and the WKS expansions with $N=14, m=7, \theta=\pi / 7$, and $h=1 / 2$.

TABLE 5.3

Approximation of the function at $\left(x_{\ell_{1}}, y_{\ell_{2}}, y_{\ell_{3}}\right):=\left(\left(\ell_{1}+1 / 2\right) h,\left(\ell_{2}+1 / 2\right) h,\left(\ell_{3}+1 / 2\right) h\right)$.

\begin{tabular}{lcc}
$\left(x_{\ell_{1}}, y_{\ell_{2}}, y_{\ell_{3}}\right)$ & \multicolumn{2}{c}{ Absolute error for the expansions $(1.2)$ and $(3.1)$} \\
\cline { 2 - 3 } & $\left|\mathfrak{T}_{14}\left(x_{\ell_{1}}, y_{\ell_{2}}, y_{\ell_{3}}\right)\right|$ & $\left|T_{14}\left(x_{\ell_{1}}, y_{\ell_{2}}, y_{\ell_{3}}\right)\right|$ \\
\hline$\left(x_{0}, y_{0}, z_{0}\right)$ & 0.000123302 & $1.23608 \times 10^{-9}$ \\
$\left(x_{1}, y_{1}, z_{1}\right)$ & 0.000395412 & $4.12706 \times 10^{-9}$ \\
$\left(x_{2}, y_{2}, z_{2}\right)$ & 0.000661489 & $7.84735 \times 10^{-8}$ \\
$\left(x_{3}, y_{3}, z_{3}\right)$ & 0.000643725 & $2.31930 \times 10^{-7}$ \\
$\left(x_{4}, y_{4}, z_{4}\right)$ & 0.000019694 & $2.91510 \times 10^{-6}$
\end{tabular}

\section{REFERENCES}

[1] M. H. ANNABy AND R. M. AshaRABI, Truncation, amplitude, and jitter errors on $\mathbb{R}$ for sampling series derivatives, J. Approx. Theory, 163 (2011), pp. 336-362.

[2] - Error estimates associated with sampling series of the linear canonical transforms, IMA J. Numer. Anal., 35 (2015), pp. 931-946.

[3] - Bounds for truncation and perturbation errors of nonuniform sampling series, BIT, 56 (2016), pp. 807-832.

[4] - Derivative sampling expansions for the linear canonical transform: convergence and error analysis, J. Comput. Math., 37 (2019), pp. 431-446.

[5] M. H. ANnAby AND M. M. Tharwat, On the computation of the eigenvalues of Dirac systems, Calcolo, 49 (2012), pp. 221-240.

[6] R. M. ASHARABI AND H. S. AL-ABBAS, Truncation error estimates for generalized Hermite sampling, Numer. Algorithms, 74 (2017), pp. 481-497.

[7] R. M. Asharabi And A. M. Al-Hayzea, Double sampling derivatives and truncation error estimates, Appl. Math. J. Chinese Univ. Ser. B, 33 (2018), pp. 209-224.

[8] P. L. BUTZER, A survey of the Whittaker-Shannon sampling theorem and some of its extensions, J. Math. Res. Exposition, 3 (1983), pp. 185-212.

[9] P. L. BUtZER AND W. SPLETTSTÖSSER, On quantization, truncation and jitter errors in the sampling theorem and its generalizations, Signal Process., 2 (1980), pp. 101-112.

[10] P. L. ButZER, W. SPLETTSTÖSSER, AND R. L. STENS, The sampling theorem and linear prediction in signal analysis, Jahresber. Deutsch. Math.-Verein., 90 (1988), pp. 1-70.

[11] B. Chanane, Computing the eigenvalues of singular Sturm-Liouville problems using the regularized sampling method, Appl. Math. Comput., 184 (2007), pp. 972-978. 
[12] C. H. Edwards, Advanced Calculus of Several Variables, Academic Press, New York, 1973.

[13] G. S. FANG AND Y. W. LI, Multidimensional sampling theorem of Hermite type and estimates for aliasing error on Sobolev classes, Chinese Ann. Math. Ser. A, 27 (2006), pp. 217-230.

[14] R. GERVAIS, Q. I. RAHMAN, AND G. SCHMEISSER, A bandlimited function simulating a duration-limited one, in Anniversary Volume on Approximation Theory and Functional Analysis, P. L. Butzer, R. L. Stens, and B. Sz.-Nagy, eds., Vol. 65 of Internat. Schriftenreihe Numer. Math., Birkhäuser, Basel, 1984, pp. 355-362.

[15] R. P. Gosselin, On the $L^{p}$ theory of cardinal series, Ann. of Math. (2), 78 (1963), pp. 567-581.

[16] J. R. Higgins, Sampling Theory in Fourier and Signal Analysis Foundations, Oxford University Press, Oxford, 1996.

[17] D. JAGERMAN, Bounds for truncation error of the sampling expansion, SIAM J. Appl. Math., 14 (1966), pp. 714-723.

[18] L. JINGFAN AND F. GENSUN, On uniform truncation error bounds and aliasing error for multidimensional sampling expansion, Sampl. Theory Signal Image Process., 2 (2003), pp. 103-115.

[19] X. M. LI, Uniform bounds for sampling expansions, J. Approx. Theory, 93 (1998), pp. 100-113.

[20] J. LONG AND G. FANG, On truncation error bound for multidimensional sampling expansion Laplace transform, Anal. Theory Appl., 20 (2004), pp. 52-57.

[21] S. M. NIKOL'SKII, Approximation of Functions of Several Variables and Imbedding Theorems, Springer, Berlin, 1975

[22] E. PARZEN, A simple proof and some extensions of sampling theorems, Technical Report 7, Dept. of Statistics, Stanford University, Stanford, 1956.

[23] D. P. PETERSEN AND D. MidDleton, Sampling and reconstruction of wave-number-limited functions in $N$-dimensional Euclidean spaces, Information and Control, 5 (1962), pp. 279-323.

[24] J. J. WANG AND G. S. FANG, A multidimensional sampling theorem and an estimate of the aliasing error, Acta Math. Appl. Sinica, 19 (1996), pp. 481-488.

[25] P. YE, Error bounds for multidimensional Whittaker-Shannon sampling expansion, Proc. 2011 International Conference on Multimedia and Signal Processing, 2011, IEEE Computer Society, Washington, DC, pp. 33-36.

[26] P. YE AND Y. HAN, Uniform bounds of aliasing and truncated errors in sampling series of functions from anisotropic Besov class, Abstr. Appl. Anal., (2013), Art. ID 154637, 9 pages.

[27] P. Ye AND Z. Song, Truncation and aliasing errors for Whittaker-Kotelinkov-Shannon sampling expanion, Appl. Math. J. Chinese Univ. Ser B, 27 (2012), pp. 412-418. 